\title{
TÉCNICAS PARA REDUÇÃO DA ORDEM DE SISTEMAS DINÂMICOS NA BASE MODAL
}

\author{
Gabriel P. R. Maciel ${ }^{1}$, Roberto Spinola barbosa ${ }^{1}$ \\ ${ }^{1}$ Escola Politécnica da USP \\ E-mails: ga.pe.01@gmail.com, spinola@usp.br
}

\section{RESUMO}

Técnicas para redução da ordem de modelos na base modal foram implementadas com o objetivo de estudar como o erro, associado ao processo de redução, é influenciado pelo número de modos truncados e pelo critério de seleção de modos a serem eliminados. Neste trabalho, são apresentados diferentes critérios para seleção dos modos a serem truncados, a interpretação de cada critério também é detalhada de maneira qualitativa para prever o comportamento do modelo reduzido. Um modelo de veículo completo com 7 graus de liberdade para estudo de dinâmica vertical foi usado como exemplo e modelos reduzidos foram calculados a partir do mesmo. Para a comparação de desempenho entre modelos reduzidos, são apresentadas métricas que facilitam a avaliação objetiva das respostas.

\section{INTRODUÇÃO}

Com o advento da tecnologia em computadores, o poder de processamento e armazenamento computacional aumentou consideravelmente nas últimas décadas. Junto a esta tendência, observase a demanda pelo aumento da complexidade de modelos numéricos, o que pode ser prejudicial em muitas aplicações. Además, é sabido que o custo computacional e o tempo de préprocessamento de modelos de sistemas dinâmicos ainda é um fator importante em processos que envolvam simulação em tempo real, otimização, processamento embarcado, ciclos de projeto com prazos de curta duração, projeto de controladores, estudo da estabilidade e robustez de sistemas. Para contornar este problema, esforços tem sido feitos no desenvolvimento de métodos para calcular modelos de ordem reduzida que reproduzam as principais características dinâmicas de um modelo de alta ordem, exigindo menor custo computacional e capacidade de memória [5].

Portanto neste trabalho, os autores expõem uma metodologia de redução da ordem de sistemas por truncamento na base modal. Neste processo, o critério de escolha dos modos normais que serão truncados do modelo de alta ordem é um fator determinante para que o modelo reduzido reproduza corretamente o comportamento dinâmico do modelo completo.

Um modelo de veículo completo com 7 graus de liberdade para estudo de dinâmica vertical foi construído para representar um modelo de alta ordem. Foram calculados três modelos de ordem reduzida, para cada modelo foi utilizado um critério diferente de seleção dos modos a serem truncados. Posteriormente os resultados dos modelos reduzidos foram comparados aos do 
modelo completo utilizando métricas que favorecem a avaliação objetiva de seus desempenhos.

\section{INFORMAÇÕES GERAIS}

Considerando um sistema dinâmico, linear, contínuo, invariante no tempo, assintoticamente estável, $[A, B, C, D]$ em sua realização mínima, com a seguinte representação em espaço de estados:

$$
\dot{x}(t)=A x(t)+B u(t) \quad y(t)=C x(t)+D u(t)
$$

Onde $x(t) \in \mathbb{R}^{n}, u(t) \in \mathbb{R}^{n_{u}}, y(t) \in \mathbb{R}^{n_{y}}$ são respectivamente o vetor de estados, vetor de entrada e vetor de saída. Este sistema é chamado de completo e considerado, para fins de redução, como um sistema de alta ordem.

Deseja-se encontrar uma aproximação de ordem reduzida $\left[A_{r}, B_{r}, C_{r}, D_{r}\right]$, eliminando-se $r$ graus de liberdade do sistema completo e cuja ordem seja $n_{r}=n-r$, com $x_{r} \in \mathbb{R}^{n_{r}}$. O sistema de ordem reduzida deve ser capaz de reproduzir aproximadamente o comportamento dinâmico do sistema original.

$$
\dot{x}_{r}(t)=A_{r} x_{r}(t)+B_{r} u(t) \quad y_{r}(t)=C_{r} x_{r}(t)+D_{r} u(t)
$$

O sistema completo pode ser particionado em duas parcelas, chamadas de graus de liberdade (GDL) mais dominantes e menos dominantes, $x_{1}$ e $x_{2}$ respectivamente.

$$
\left\{\begin{array}{c}
\dot{x}_{1} \\
\dot{x}_{2} \\
\hline y
\end{array}\right\}=\left[\begin{array}{cc|c}
A_{11} & A_{12} & B_{1} \\
A_{21} & A_{22} & B_{2} \\
\hline C_{1} & C_{2} & D
\end{array}\right]\left\{\begin{array}{c}
x_{1} \\
x_{2} \\
\hline u
\end{array}\right\}
$$

Neste trabalho, será considerado que o sistema reduzido é obtido através do truncamento dos GDL menos dominantes, ou seja, $x_{2}=0$. Portanto $A_{r}=A_{11}, B_{r}=B_{1}, C_{r}=C_{1}$ and $D_{r}=D$.

O sistema que descreve o comportamento dinâmico do erro $e(t)=y(t)-y_{r}(t)$ entre os sistemas completo e reduzido é formado pelas matrizes $\left[A_{e}, B_{e}, C_{e}, D_{e}\right]$ tal que:

$$
\left\{\begin{array}{c}
\dot{x}_{1} \\
\dot{x}_{2} \\
\dot{x}_{r} \\
\hline e
\end{array}\right\}=\left[\begin{array}{ccc|c}
A_{11} & A_{12} & 0 & B_{1} \\
A_{21} & A_{22} & 0 & B_{2} \\
0 & 0 & A_{11} & B_{1} \\
\hline C_{1} & C_{2} & -C_{1} & 0
\end{array}\right]\left\{\begin{array}{c}
x_{1} \\
x_{2} \\
x_{r} \\
\hline u
\end{array}\right\}
$$

Onde $\hat{x}=\left\{x_{1} ; x_{2} ; x_{r}\right\}$ é o vetor de estados do sistema na Eq. 4 e $A_{e} \in \mathbb{R}^{\left(n+n_{r}\right) \mathrm{x}\left(n+n_{r}\right)}$.

O erro quadrático é uma maneira de contabilizar em uma única curva a resposta temporal o vetor $e(t)=\left(e_{1}(t), e_{2}(t), \ldots, e_{n_{y}}(t)\right)^{T}$ do sistema reduzido, o que falicita a avaliação de desempenho do mesmo. A utilização do erro quadrático torna a comparação entre vários modelos mais fácil e objetiva pois reduz a quantidade de dados a serem comparados.

$$
e_{q}(t)=e_{1}^{2}(t)+e_{2}^{2}(t)+\cdots+e_{n_{y}}^{2}(t)+=e^{T}(t) e(t)
$$

Uma outra maneira de quantificar a diferença entre duas respostas transientes é a norma $\mathcal{L}_{2}$ do erro $e(t)$. Diferente do erro quadrático, a norma $\mathcal{L}_{2}$ do erro quantifica a diferença entre as 
respostas dos sistemas completo e reduzido em apenas um valor escalar. Isso possibilita a comparação do desempenho de diferentes modelos através da mera comparação entre números reais.

A norma $\mathcal{L}_{2}$ do erro $e(t)$ no intervalo $[0, t]$ é definida por:

$$
\left\|y(t)-y_{r}(t)\right\|_{\mathcal{L}_{2}[0, t]}=\|e(t)\|_{\mathcal{L}_{2}[0, t]}=\left(\int_{0}^{t} e^{T}(\tau) e(\tau) d \tau\right)^{\frac{1}{2}}
$$

\subsection{Decomposição em modos normais}

Dado um sistema dinâmico linear com $n_{0}$ coordenadas generalizadas, do tipo

$$
M \ddot{q}+C_{a} \dot{q}+K q=F u
$$

Sendo $q \in \mathbb{R}^{n_{0}}$ é o vetor de coordenadas generalizadas do sistema e as matrizes $M, C_{a}$ e $K$ são quadradas de ordem $n_{0}$. Também assume-se que $M$ é positiva definida, portanto existe a matriz inversa $M^{-1}$.

Consideraremos um caso genérico onde o amortecimento do sistema em questão é dito não proporcional, ou seja, a matriz de amortecimento $C_{a}$ não pode ser escrita como uma combinação linear das matrizes $M$ e $K$.

Deseja-se projetar o sistema em uma base ortonormal de autovetores, que posteriormente será interpretada como uma sobreposição de modos de vibração do sistema. Definem-se como modos de vibração síncronos de um sistema os padrões de movimento oscilatório em que coordenadas generalizadas possuem relações de proporcionalidade constantes.

Consideraremos o sistema não excitado na forma

$$
M \ddot{q}+C_{a} \dot{q}+K q=0
$$

Através da mudança de base $x=\left(q^{T}, \dot{q}^{T}\right)^{T}$, o sistema de segunda ordem com $n_{0}$ equações diferenciais ordinárias pode ser escrito como um sistema de primeira ordem com $n=2 n_{0}$ equações. Desta maneira, a equação 8 pode ser escrita na forma

$$
\left\{\begin{array}{c}
\dot{q} \\
\ddot{q}
\end{array}\right\}=\left[\begin{array}{cc}
0 & I \\
-M^{-1} K & -M^{-1} C_{a}
\end{array}\right] \cdot\left\{\begin{array}{l}
q \\
\dot{q}
\end{array}\right\}
$$

Onde $x \in \mathbb{R}^{2 n_{0}}$ é um vetor que contém deslocamentos e velocidades generalizadas do sistema. Desta forma, podemos escrever sinteticamente

$$
\dot{x}=A x
$$

Desejamos agora encontrar uma base ortonormal a fim de realizar a decomposição do sistema em modos de vibrar, desta maneira a solução da equação 10 é da forma

$$
x(t)=e^{s t} z
$$

onde s é um escalar e $z \in \mathbb{R}^{n}$. Substituindo-se 11 em 10, obtém-se

$$
A z_{k}=\lambda_{k} z_{k} \quad k=1,2, \ldots, n_{0}
$$


A partir da equação 12, pode-se concluir que $z_{k}$ é o k-ésimo autovetor direito de A e $\lambda_{k}$ é seu autovalor associado. Neste trabalho, serão considerados apenas casos onde os autovalores do sistema são distintos, ou seja, não existe $\lambda_{i}=\lambda_{j}$ para $i \neq j$.

Podemos observar que a equação 12 possui $n$ soluções não triviais $\lambda_{k}$ que são autovalores da matriz $A$. Pode-se provar [10] que estas soluções podem ser reais e/ou pares pares complexos conjugados. Consequentemente, seus autovetores correspondentes são respectivamente reais e complexos conjugados. Neste último caso, temos:

$$
\begin{aligned}
& A z_{k}=\lambda_{k} z_{k} \\
& A \bar{z}_{k}=\bar{\lambda}_{k} \bar{z}_{k}
\end{aligned}
$$

onde

$$
z_{k}=u_{k}+i v_{k} \quad \text { e } \quad \bar{z}_{k}=u_{k}-i v_{k}
$$

Sem que exista perda de generalização, a partir deste ponto as equações serão apresentadas considerando que todos os autovalores do sistema são complexos conjugados. O caso particular para autovalores reais é deduzido de maneira análoga.

Seja uma matriz $\Phi$ de ordem $n$, cujas colunas são as componentes reais e complexas de todos os autovetores direitos de $A$

$$
\Phi=\left[\begin{array}{llllllll}
u_{1} & v_{1} & \ldots & u_{k} & v_{k} & \ldots & u_{n_{0}} & v_{n_{0}}
\end{array}\right]
$$

Onde os vetores $u_{k}$ e $v_{k}$ são chamados de formas modais que compõem o k-ésimo modo normal do sistema.

Pode-se demonstrar [4] que a matriz $\Phi$ é inversível e que é uma matriz de mudança de base do tipo

$$
x=\Phi w
$$

Onde $\omega$ são chamadas de coordenadas modais do sistema. Aplicando-se a transformação descrita em 16 ao sistema em espaço de estados da equação 1, temos:

$$
\left\{\begin{array}{c}
\Phi \dot{w}=A \Phi w+B u \\
y=C \Phi w+D u
\end{array}\right.
$$

onde $B=M^{-1} F$. Assim, podemos definir o sistema $[\tilde{A}, \tilde{B}, \tilde{C}, \tilde{D}]$ na base modal, com:

$$
\begin{gathered}
\widetilde{A}=\Phi^{-1} A \Phi \\
\widetilde{B}=\Phi^{-1} B \\
\widetilde{C}=C \Phi \\
\widetilde{D}=D
\end{gathered}
$$

e consequentemente

$$
\left\{\begin{array}{c}
\dot{w} \\
\hline y
\end{array}\right\}=\left[\begin{array}{c|c}
\Phi^{-1} A \Phi & \Phi^{-1} B \\
\hline C \Phi & D
\end{array}\right] \cdot\left\{\begin{array}{c}
w \\
\hline u
\end{array}\right\}
$$


Sendo assim, a matriz $\widetilde{A}$, de ordem $n=2 n_{0}$, é composta por blocos $2 \times 2$ correspondentes aos pares de autovalores complexos de $A$ (e blocos 1x1 para o caso de autovalores reais).

Desta maneira, o sistema na base modal pode ser escrito como:

$$
\left[\begin{array}{c|c}
\tilde{A} & \tilde{B} \\
\hline \tilde{C} & \tilde{D}
\end{array}\right]=\left[\begin{array}{ccccc|ccc}
\alpha_{1} & \beta_{1} & & & 0 & \tilde{b}_{1,1} & \ldots & \tilde{b}_{1, n_{u}} \\
-\beta_{1} & \alpha_{1} & & & & \tilde{b}_{2,1} & \ldots & \tilde{b}_{2, n_{u}} \\
& & \ddots & & & \vdots & & \\
& & & \alpha_{n_{0}} & \beta_{n_{0}} & \tilde{b}_{n, 1}^{\prime} & & \tilde{b}_{n, n_{u}}^{\prime} \\
0 & & & -\beta_{n_{0}} & \alpha_{n_{0}} & \tilde{b}_{n, 1}^{\prime} & & \tilde{b}_{n, n_{u}}^{\prime} \\
\hline \tilde{c}_{1,1} & \tilde{c}_{1,2} & \ldots & \tilde{c}_{1, n-1} & \tilde{c}_{1, n} & d_{1,1} & \cdots & d_{1, n_{u}} \\
\vdots & & & & & \vdots & & \\
\tilde{c}_{n_{y}, 1} & \tilde{c}_{n_{y}, 2} & \cdots & \tilde{c}_{n_{y}, n-1} & \tilde{c}_{n_{y}, n} & d_{n_{y}, 1} & \cdots & d_{n_{y}, n_{u}}
\end{array}\right]
$$

A equação 19 pode ser desacoplada em subsistemas menores de ordem 2, como mostrados na equação 21. Cada subsistema descreve a dinâmica de um modo normal de vibração do sistema completo. A resposta $y_{k}$ é chamada de participação modal do modo $k$ na resposta do sistema.

$$
\left\{\begin{array}{c}
\dot{w}_{2 k-1} \\
\dot{w}_{2 k} \\
\hline y_{k}
\end{array}\right\}=\left[\begin{array}{cc|ccc}
\alpha_{k} & \beta_{k} & \tilde{b}_{2 k-1,1} & \cdots & \tilde{b}_{2 k-1, n_{u}} \\
-\beta_{k} & \alpha_{k} & \tilde{b}_{2 k, 1} & \cdots & \tilde{b}_{2 k, n_{u}} \\
\hline \tilde{c}_{1,2 k-1} & \tilde{c}_{1,2 k} & 0 & \cdots & 0 \\
\vdots & & \vdots & & \\
\tilde{c}_{n_{y}, 2 k-1} & \tilde{c}_{n_{y}, 2 k} & 0 & \cdots & 0
\end{array}\right]\left\{\begin{array}{c}
w_{2 k-1} \\
w_{2 k} \\
u u
\end{array}\right\}
$$

Vale lembrar que o vetor $y(t)$ é resultado da soma das participações modais $y_{k}(t)$ com o termo Du:

$$
y(t)=\sum_{k=1}^{n_{0}} y_{k}(t)+D u(t)
$$

\section{CRITÉRIOS DE SELEÇÃO}

Neste item serão apresentados e discutidos critérios para a seleção dos modos nomais que são bons quandidatos a eliminação. Estes critérios colocam as variáveis do sistema completo em ordem das mais importantes para as menos importantes, de maneira que as $r$ últimas da lista serão eliminadas durante o processo de redução da ordem do sistema.

Neste trabalho, são estabelecidos parâmetros seleção tais que modos com menores valores atribuídos ao parâmetro são bons candidatos à eliminação.

\subsection{Critério de seleção baseado na frequência natural (FN)}

Um critério de seleção bastante usado para a base modal é descrito em [1], onde o parâmetro $P_{\omega_{n}}^{(k)}$ é o inverso da frequência natural $\omega_{n k}$ do k-ésimo modo de vibrar do sistema.

$$
P_{\omega_{n}}^{(k)}=\frac{1}{\omega_{n k}}=\frac{1}{\sqrt{\alpha_{k}^{2}+\beta_{k}^{2}}}
$$

Uma interpretação geométrica para o parâmetro de seleção $P_{\omega_{n}}$ é que os polos candidatos à eliminação estão fora de circunferência de raio $\omega_{n 0}$ e com centro em $(0,0)$, conforme indicado pela figura 1. 


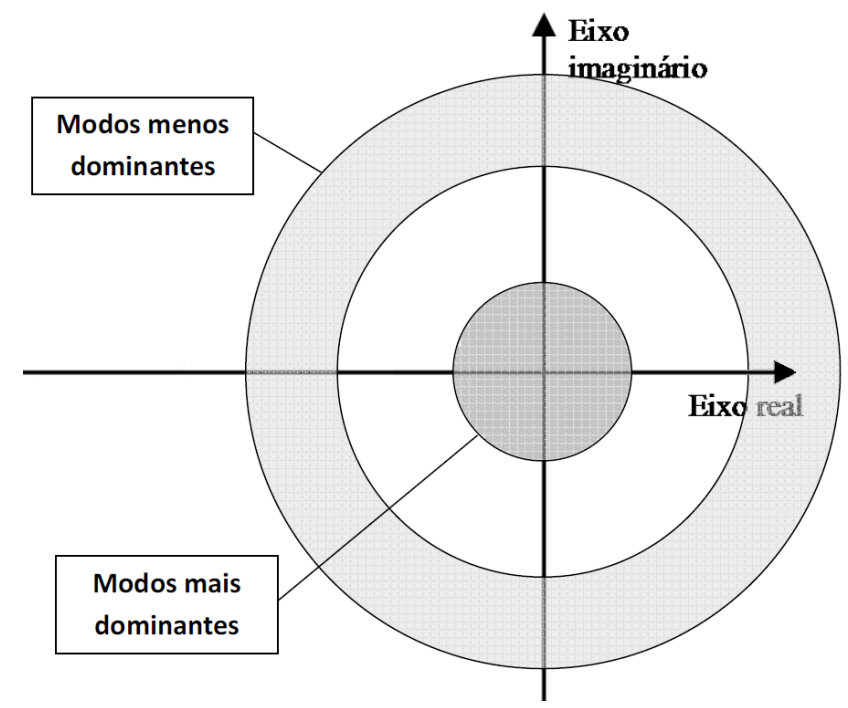

Figura 1: Critério de seleção baseado na frequência natural dos autovalores

Como com a utilização deste critério, os modos de menor frequência natural são preservados, ele é aconselhado para situações onde deseja-se priorizar preservação da resposta do sistema quando é submetido à excitações periódicas de baixas frequências.

Uma desvantagem deste método é que modos com baixo fator de amortecimento e maiores frequências naturais (que seriam bons candidatos a serem eliminados segundo este critério) podem ter contribuição importante na resposta transiente do sistema. Portanto ao se aplicar este critério, a similitude da resposta transitória do sistema reduzido pode ser comprometida quando comparada ao modelo completo.

\subsection{Critério de seleção baseado no amortecimento e frequência natural (AFN)}

Este critério, descrito por [7], para seleção dos modos candidatos à eliminação, é baseado no fator de amortecimento $\zeta$ e frequência não amortecida $\omega_{n}$ de cada modo de vibrar.

Sabendo que o produto $\omega_{n} \zeta$ está diretamente relacionado com a potência dissipada do modo, este critério que penaliza modos de alto fator de amortecimento e alta frequência natural. $\mathrm{O}$ parâmetro de seleção $P_{a f}^{(k)}$ para um modo é o inverso do produto do fator de amortecimento $\zeta$ do modo com sua frequência natural $\omega_{n}$ correspondente.

$$
P_{a f}^{(k)}=\frac{1}{\omega_{n k} \zeta_{k}}=\frac{1}{-R e\left(\lambda_{k}\right)}=\frac{1}{-\alpha_{k}}
$$

Também é sabido que o produto do amortecimento de um modo com sua frequência natural é igual ao módulo da parte real do autovalor correspondente. Portanto o parâmetro de seleção $P_{a f}^{(k)}$ também pode ser calculado através do inverso da parte real do autovalor correspondente ao modo normal, como mostrado na equação 24 .

A figura 2 mostra uma representação gráfica dos modos mais dominantes e menos dominantes do sistema (estes últimos candidados à eliminação).

Outro ponto importante para salinetar é que, para uma entrada degrau, o tempo de acomodação $t_{s}$ do k-ésimo modo normal é [8]: 


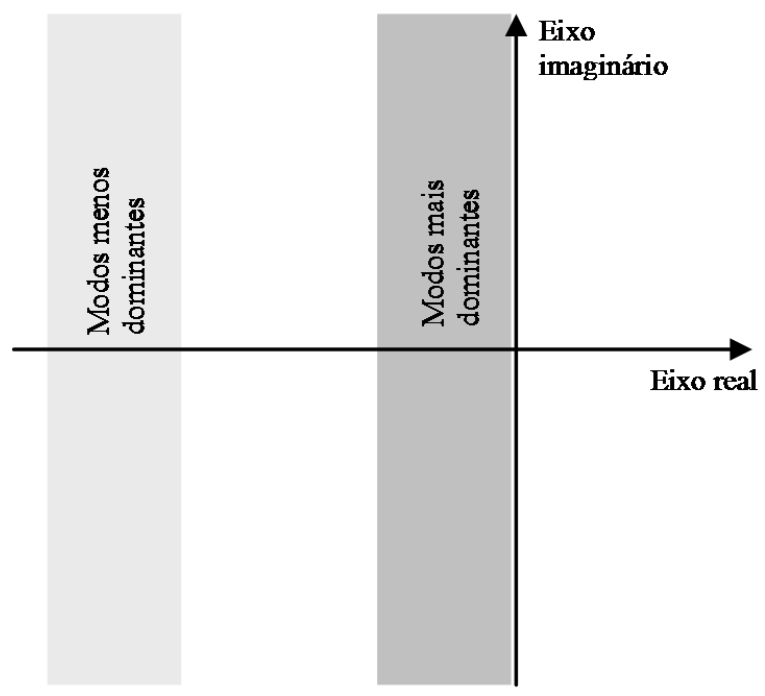

Figura 2: Critério de seleção baseado na parte real dos autovalores

$$
t_{s k}=\frac{4}{\omega_{n k} \zeta_{k}}
$$

Portanto segundo este critério, os modos que são bons candidatos a serem eliminados tem tempo de acomodação pequeno. Ou seja, estabilizam mais rapidamente na condição de estado estável que outros modos. Isso reforça o conceito de que este critério preserva modos com maior contribuição para a resposta transiente do sistema.

Conclui-se que este critério é aconselhado para situações onde deseja-se priozizar a preservação do comportamento transiente do sistema, como por exemplo quando submetido à excitações não periódicas.

\subsection{Critério de seleção baseado em normas Hankel (NH)}

Partindo da informação de que as normas Hankel de um sistema dinâmico indicam o quanto este sistema é simultaneamente controlável e observável, pode-se estabelecer um critério que selecione os modos normais menos controláveis e observáveis para serem eliminados [3], [9].

As normas Hankel $\gamma_{i}$ de um sistema dinâmico são a raiz dos autovalores do produto entre os gramianos infinitos de controlabilidade $L_{c}$ e observabilidade $L_{o}$, calculadas conforme equação 26.

$$
\gamma_{i}=\sqrt{\lambda_{i}\left(L_{c} L_{o}\right)}
$$

Onde $\lambda_{i}$ é o i-ésimo autovalor do produto $L_{c} L_{o}$.

Por sua vez, os gramianos infinitos de controlabilidade e observabilidade são calculados segundo as equações matriciais de Lyapunov:

$$
\begin{aligned}
& A L_{c}+L_{c} A^{T}+B B^{T}=0 \\
& A^{T} L_{o}+L_{o} A+C^{T} C=0
\end{aligned}
$$


Neste critério, descrito por Varga em [11], o sistema completo projetado na base modal é separado em subsistemas desacoplados que descrevem a dinâmica dos modos normais, como na equação 21. As normas Hankel destes subsistemas são calculadas e a maior norma de cada modo é usada como critério de seleção.

$$
P_{\gamma m}^{(k)}=\max _{i} \gamma_{i}^{(k)} \quad k=1,2, \ldots, n
$$

Onde $\gamma_{i}^{(k)}$ são as normas Hankel do k-ésimo subsistema e $P_{\gamma m}^{(k)}$ é o parâmetro de seleção baseado na controlabilidade e observabilidade dos modos normais. Os valores de $\gamma_{i}^{(k)}$ são encontrados ao aplicar as equações 26 e 27 em cada um dos sistemas descritos na equação 21.

Os autores se utilizarão de um abuso de linguagem e neste trabalho as normas Hankel calculadas a partir dos sistemas desacoplados que descrevem a dinâmica dos modos normais serão chamadas de normas Hankel dos modos normais.

\section{ESTUDO DE CASO}

Um modelo de veículo completo, com 4 rodas e com 7 graus de liberdade, para estudo de dinâmica vertical, como descrito em [6], foi construído para representar um modelo de alta ordem. Neste exemplo, foram usados parâmetros que representam um carro de passeio com suspensões independentes e pneus.

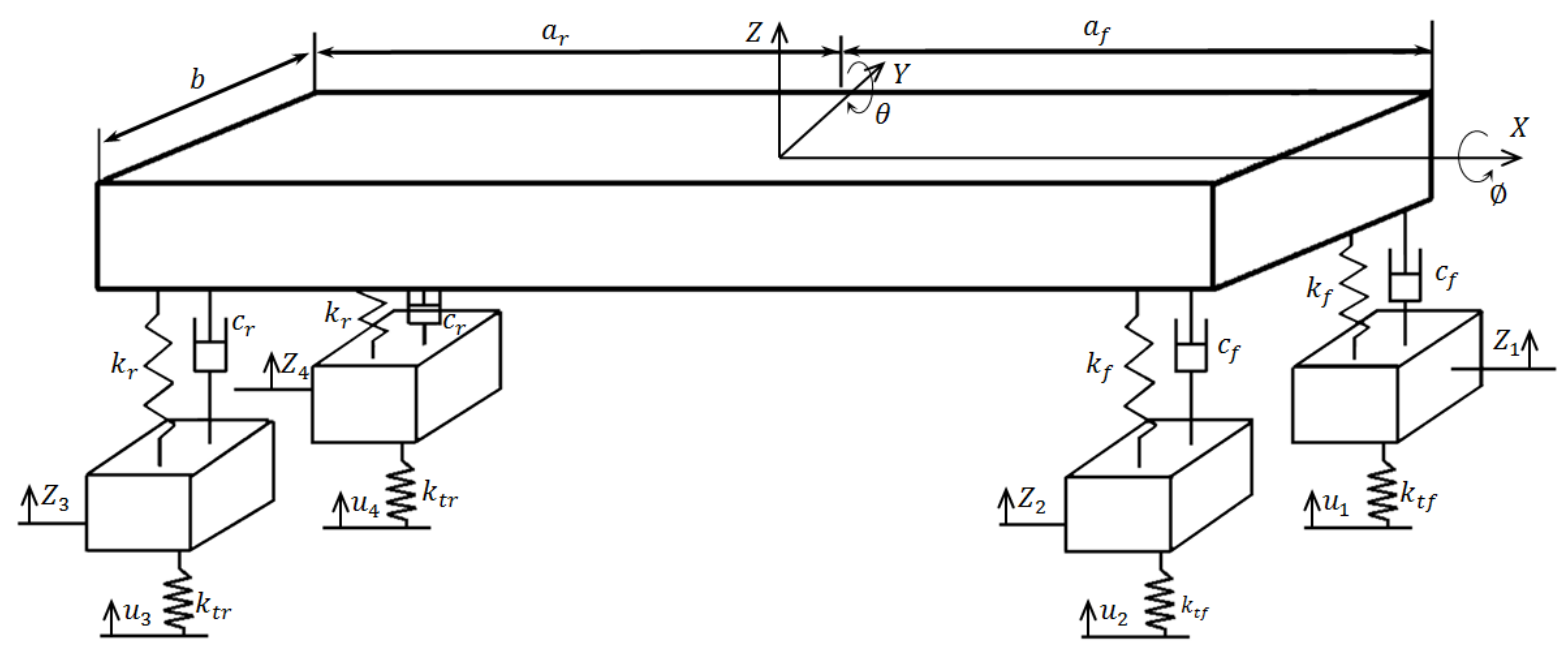

Figura 3: Modelo de veículo completo com 7 graus de liberdade.

As equações de movimento serão deduzidas utilizando técnica de sistemas multicorpos [2]. Os graus de liberdade do sistema são:

- Z : Deslocamento vertical do chassi

- $\phi$ : ângulo de rolagem do chassi

- $\theta$ : ângulo de arfagem do chassi 
- $Z_{1}$ : Deslocamento vertical do centro da roda dianteira esquerda

- $Z_{2}$ : Deslocamento vertical do centro da roda dianteira direita

- $Z_{3}$ : Deslocamento vertical do centro da roda traseira direita

- $Z_{4}$ : Deslocamento vertical do centro da roda traseira esquerda

O vetor $q$ define graus de liberdade, ou coordenadas generalizadas, do sistema pode ser escrito na forma:

$$
q=\left\{\begin{array}{lllllll}
Z & \phi & \theta & Z_{1} & Z_{2} & Z_{3} & Z_{4}
\end{array}\right\}^{T}
$$

As equações que definem as energias cinética e potencial do sistema são mostradas respectivamente em 30 e 31.

$$
\begin{gathered}
T=\frac{1}{2} m_{c} \dot{Z}^{2}+\frac{1}{2} I_{x} \dot{\phi}^{2}+\frac{1}{2} I_{y} \dot{\theta}^{2}+\frac{1}{2} m_{f}\left(\dot{Z}_{1}^{2}+\dot{Z}_{2}^{2}\right)+\frac{1}{2} m_{r}\left(\dot{Z}_{3}^{2}+\dot{Z}_{4}^{2}\right) \\
V=\frac{1}{2} k_{f}\left(Z-Z_{1}+b \phi-a_{f} \theta\right)^{2}+\frac{1}{2} k_{f}\left(Z-Z_{2}-b \phi-a_{f} \theta\right)^{2}+ \\
+\frac{1}{2} k_{r}\left(Z-Z_{3}-b \phi+a_{r} \theta\right)^{2}+\frac{1}{2} k_{r}\left(Z-Z_{4}+b \phi+a_{f} \theta\right)^{2}+\frac{1}{2} k_{t f}\left(Z_{1}-Z_{r 1}\right)^{2}+ \\
+\frac{1}{2} k_{t f}\left(Z_{2}-Z_{r 2}\right)^{2}+\frac{1}{2} k_{t r}\left(Z_{3}-Z_{r 3}\right)^{2}+\frac{1}{2} k_{t r}\left(Z_{4}-Z_{r 4}\right)^{2}
\end{gathered}
$$

Utilizando a chamada equação de Lagrange [2]

$$
\frac{d}{d t}\left(\frac{\partial L}{\partial \dot{q}_{i}}\right)-\frac{\partial L}{\partial q_{i}}=Q_{i}^{\prime}
$$

foram calculadas as matrizes de massa, amortecimento e rigidez do sistema ( $M, C_{a}$ e $K$ respectivamente).

$$
M=\left[\begin{array}{ccccccc}
m_{c} & 0 & 0 & 0 & 0 & 0 & 0 \\
0 & I_{x} & 0 & 0 & 0 & 0 & 0 \\
0 & 0 & I_{y} & 0 & 0 & 0 & 0 \\
0 & 0 & 0 & m_{f} & 0 & 0 & 0 \\
0 & 0 & 0 & 0 & m_{f} & 0 & 0 \\
0 & 0 & 0 & 0 & 0 & m_{t} & 0 \\
0 & 0 & 0 & 0 & 0 & 0 & m_{t}
\end{array}\right]
$$




$$
\begin{aligned}
& C_{a}=\left[\begin{array}{cccc}
2\left(c_{f}+c_{r}\right) & 0 & 2\left(-a_{f} c_{f}+a_{r} c_{r}\right) & -c_{f} \\
0 & 2 b^{2}\left(c_{f}+c_{r}\right) & 0 & -b c_{f} \\
2\left(-a_{f} c_{f}+a_{r} c_{r}\right) & 0 & 2\left(a_{f}^{2} c_{f}+a_{r}^{2} c_{r}\right) & a_{f} c_{f} \\
-c_{f} & -b c_{f} & a_{f} c_{f} & c_{f} \\
-c_{f} & b c_{f} & a_{f} c_{f} & 0 \\
-c_{r} & b c_{r} & -a_{r} c_{r} & 0 \\
-c_{r} & -b c_{r} & -a_{r} c_{r} & 0
\end{array}\right. \\
& \left.\begin{array}{ccc}
-c_{f} & -c_{r} & -c_{r} \\
b c_{f} & b c_{r} & -b c_{r} \\
a_{f} c_{f} & -a_{r} c_{r} & -a_{r} c_{r} \\
0 & 0 & 0 \\
c_{f} & 0 & 0 \\
0 & c_{r} & 0 \\
0 & 0 & c_{r}
\end{array}\right]
\end{aligned}
$$

$$
\begin{aligned}
& K=\left[\begin{array}{cccc}
2\left(k_{f}+k_{r}\right) & 0 & -2 k_{f} a_{f}+2 k_{r} a_{r} & -k_{f} \\
0 & 2 b^{2}\left(k_{f}+k_{r}\right) & 0 & -k_{f} \\
2\left(a_{r} k_{r}-a_{f} k_{f}\right) & 0 & 2\left(a_{r}^{2} k_{r}+a_{f}^{2} k_{f}\right) & a_{f} k_{f} \\
-k_{f} & -b k_{f} & a_{f} k_{f} & k_{f}+k_{t f} \\
-k_{f} & b k_{f} & a_{f} k_{f} & 0 \\
-k_{r} & b k_{r} & -a_{r} k_{r} & 0 \\
-k_{r} & -b k_{r} & -a_{r} k_{r} & 0
\end{array}\right. \\
& \left.\begin{array}{ccc}
-k_{f} & -k_{r} & -k_{r} \\
k_{f} & k_{r} & -k_{r} \\
a_{f} k_{f} & -a_{r} k_{r} & -a_{r} k_{r} \\
0 & 0 & 0 \\
k_{f}+k_{t f} & 0 & 0 \\
0 & k_{r}+k_{t r} & 0 \\
0 & 0 & k_{r}+k_{t r}
\end{array}\right]
\end{aligned}
$$

Desta maneira, pode-se escrever o modelo na forma

$$
F=\left[\begin{array}{cccc}
0 & 0 & 0 & 0 \\
0 & 0 & 0 & 0 \\
0 & 0 & 0 & 0 \\
k_{t f} & 0 & 0 & 0 \\
0 & k_{t f} & 0 & 0 \\
0 & 0 & k_{t r} & 0 \\
0 & 0 & 0 & k_{t r}
\end{array}\right]
$$

$$
M \ddot{q}+C_{a m} \dot{q}+K q=F u
$$

Os valores dos parâmetros usados para a simulação do modelo de veículo completo estão especificados na tabela 1.

O sistema também pode ser escrito na forma de espaço de estados como na equação 1 onde as respectivas matrizes [A,B,C,D] são mostradas na equação 38 . 
Tabela 1: Dados de entrada do modelo para estudo de caso

\begin{tabular}{|l|c|c|c|}
\hline Parâmetro & Variável & Unidade & Valor \\
\hline Massa do Chassi & $m_{c}$ & $\mathrm{~kg}$ & 1136 \\
\hline Momento de inércia de rolagem & $I_{x}$ & $\mathrm{~kg} \mathrm{~m} \mathrm{~m}^{2}$ & 600 \\
\hline Momento de inércia de arfagem & $I_{y}$ & $\mathrm{~kg} \mathrm{~m} \mathrm{~m}^{2}$ & 2400 \\
\hline Massa de cada roda dianteira & $m_{f}$ & $\mathrm{~kg}$ & 60 \\
\hline Massa de cada roda traseira & $m_{r}$ & $\mathrm{~kg}$ & 60 \\
\hline Rigidez da mola dianteira & $k_{f}$ & $\mathrm{~N} / \mathrm{m}$ & 20620 \\
\hline Rigidez da mola traseira & $k_{r}$ & $\mathrm{~N} / \mathrm{m}$ & 20620 \\
\hline Rigidez do amortecedor dianteiro & $c_{f}$ & $\mathrm{~N} /(\mathrm{m} / \mathrm{s})$ & 3924 \\
\hline Rigidez do amortecedor traseiro & $c_{r}$ & $\mathrm{~N} /(\mathrm{m} / \mathrm{s})$ & 2943 \\
\hline Rigidez do pneu dianteiro & $k_{t f}$ & $\mathrm{~N} / \mathrm{m}$ & 190000 \\
\hline Rigidez do pneu traseiro & $k_{t r}$ & $\mathrm{~N} / \mathrm{m}$ & 190000 \\
\hline $\begin{array}{l}\text { Distância longitudinal entre centro de massa do } \\
\text { chassi e eixo dianteiro }\end{array}$ & $a_{f}$ & $\mathrm{~m}$ & 1.15 \\
\hline $\begin{array}{l}\text { Distância longitudinal entre centro de massa do } \\
\text { chassi e eixo traseiro }\end{array}$ & $a_{r}$ & $\mathrm{~m}$ & 1.15 \\
\hline $\begin{array}{l}\text { Distância lateral entre centro de massa do chassi } \\
\text { e centros das rodas }\end{array}$ & $b$ & $\mathrm{~m}$ & 0.9 \\
\hline
\end{tabular}

$$
\begin{aligned}
A & =\left[\begin{array}{ccc}
0 & I \\
-M^{-1} K & -M^{-1} C_{a m}
\end{array}\right] \\
B & =\left[\begin{array}{c}
0 \\
M^{-1} F
\end{array}\right] \\
C & =\left[\begin{array}{cccccccccccccc}
0 & 0 & 0 & 0 & 0 & 0 & k_{t f} & 0 & 0 & 0 & 0 & 0 & 0 & 0 \\
0 & 0 & 0 & 0 & 0 & 0 & 0 & 0 & k_{t f} & 0 & 0 & 0 & 0 & 0 \\
0 & 0 & 0 & 0 & 0 & 0 & 0 & 0 & 0 & 0 & k_{t r} & 0 & 0 & 0 \\
0 & 0 & 0 & 0 & 0 & 0 & 0 & 0 & 0 & 0 & 0 & 0 & k_{t r} & 0
\end{array}\right] \\
D & =\left[\begin{array}{cccccc}
-k_{t f} & 0 & 0 & 0 \\
0 & -k_{t f} & 0 & 0 \\
0 & 0 & -k_{t r} & 0 \\
0 & 0 & 0 & -k_{t r}
\end{array}\right]
\end{aligned}
$$

As matrizes $C$ e $D$ foram construídas de maneira que os componentes da saída $y(t)=\left[y_{1}(t)\right.$, $\left.y_{2}(t), y_{3}(t), y_{4}(t)\right]^{T}=\left[F_{1}(t), F_{2}(t), F_{3}(t), F_{4}(t)\right]^{T}$ sejam respectivamente as respostas temporais das forças normais de contato nos pneus dianteiro esquerdo, dianteiro direito, traseiro direito e traseiro esquerdo.

\section{RESULTADOS}

Neste item, serão apresentadas as características modais do sistema completo, bem como a ordem na qual os critérios de seleção apresentados organizam os modos normais de acordo com sua importância. 
Posteriormente, serão construídos 3 modelos reduzidos por truncamento de modos, um para cada critério de seleção apresentado no item 2.

4.1 Características modais do modelo completo

A Figura 4 mostra o diagrama de polos e zeros do modelo completo. Os polos do sistema são indicados com o símbolo $\mathbb{X}$ e os zeros com o símbolo $\mathbb{O}$.

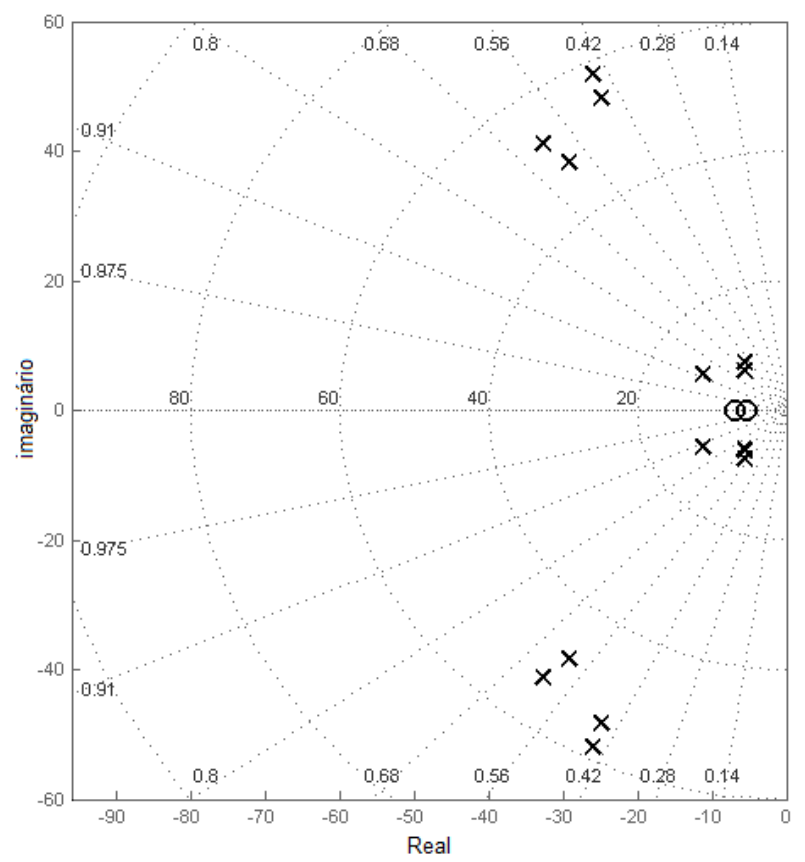

Figura 4: Diagrama de polos e zeros do sistema

A tabela 2 mostra os pólos do sistema completo organizados de maneira que a frequência natural aparece em ordem crescente. Nas colunas 3 e 4 da mesma tabela, são mostrados para cada modo, os valores da frequência natural não amortecida $\omega_{n}$ e fator de amortecimento $\zeta$ respectivamente.

Tabela 2: Propriedades dos polos do sistema

\begin{tabular}{|c|c|c|c|c|}
\hline $\begin{array}{c}\mathrm{n}^{-} \text {do } \\
\text { modo }\end{array}$ & $\begin{array}{c}\text { Parte } \\
\text { real }\end{array}$ & $\begin{array}{c}\text { Parte } \\
\text { imaginária }\end{array}$ & $\begin{array}{c}\text { Frequência } \\
\omega_{n}(\mathrm{~Hz})\end{array}$ & $\begin{array}{c}\text { Amortecimento } \\
\zeta\end{array}$ \\
\hline 1 & $-5,60$ & $\pm 6,07$ & 1,31 & 0,678 \\
\hline 2 & $-5,67$ & $\pm 7,50$ & 1,50 & 0,603 \\
\hline 3 & $-11,2$ & $\pm 5,63$ & 2,00 & 0,894 \\
\hline 4 & $-29,2$ & $\pm 38,2$ & 7,66 & 0,607 \\
\hline 5 & $-32,7$ & $\pm 41,2$ & 8,37 & 0,621 \\
\hline 6 & $-24,8$ & $\pm 48,3$ & 8,64 & 0,458 \\
\hline 7 & $-26,0$ & $\pm 52,0$ & 9,25 & 0,448 \\
\hline
\end{tabular}




\subsection{Seleção dos modos candidatos à eliminação}

Neste item serão apresentados os parâmetros de seleção para eliminação de modos normais do sistema completo. Os modos foram organizados de maneira que o parâmetro de seleção esteja em ordem decrescente, portanto modos associados a menores valores são bons candidatos à eliminação.

A tabela 3 mostra os parâmetros $P_{\omega_{n}}^{(k)}$, segundo o critério baseado na frequência natural (FN). Este parâmetro é igual ao inverso da frequência natural $\omega_{n}$ (em radianos por segundo), conforme equação 23.

Tabela 3: Ordem de importância dos modos normais segundo o critério FN

\begin{tabular}{|c|c|c|}
\hline $\begin{array}{c}\text { Importância } \\
\text { segundo critério }\end{array}$ & $\begin{array}{c}\mathrm{n}^{\underline{o}} \text { do } \\
\text { modo }\end{array}$ & $\begin{array}{c}\text { Parâmetro de } \\
\text { seleção } P_{\omega_{n}}\end{array}$ \\
\hline $1^{\underline{o}}$ & 1 & 0,1212 \\
\hline $2^{\underline{o}}$ & 2 & 0,1063 \\
\hline $3^{\underline{o}}$ & 3 & 0,0795 \\
\hline $4^{\underline{o}}$ & 4 & 0,0208 \\
\hline $5^{\underline{o}}$ & 5 & 0,0190 \\
\hline $6^{\underline{o}}$ & 6 & 0,0184 \\
\hline $7^{o}$ & 7 & 0,0172 \\
\hline
\end{tabular}

A tabela 4 mostra os parâmetros $P_{a f}^{(k)}$, segundo o critério baseado no amortecimento e frequência (AFN). Este parâmetro é igual ao inverso do produto da frequência natural $\omega_{n}(\mathrm{em} \mathrm{rad} / \mathrm{s})$ pelo fator de amortecimento $\zeta$ do mesmo modo, conforme equação 24 .

Tabela 4: Ordem de importância dos modos normais segundo o critério AFN

\begin{tabular}{|c|c|c|}
\hline $\begin{array}{c}\text { Importância } \\
\text { segundo critério }\end{array}$ & $\begin{array}{c}\mathrm{n}^{\underline{o}} \text { do } \\
\text { modo }\end{array}$ & $\begin{array}{c}\text { Parâmetro de } \\
\text { seleção } P_{a f}\end{array}$ \\
\hline $1^{o}$ & 1 & 0,1787 \\
\hline $2^{\underline{o}}$ & 2 & 0,1764 \\
\hline $3^{\underline{o}}$ & 3 & 0,0889 \\
\hline $4^{\underline{o}}$ & 6 & 0,0402 \\
\hline $5^{\underline{o}}$ & 7 & 0,0384 \\
\hline $6^{\underline{o}}$ & 4 & 0,0342 \\
\hline $7^{o}$ & 5 & 0,0306 \\
\hline
\end{tabular}

A tabela 5 mostra as normas Hankel dos modos normais. Como o sistema completo possui autovalores complexos e distintos, cada um dos sistemas desacoplados que descrevem a dinâmica dos modos normais tem ordem 2 e portanto duas normas Hankel cada. As normas Hankel dos modos normais foram organizadas esendo $\gamma_{1}$ a maior de cada par e $\gamma_{2}$ a menor. 
Tabela 5: Normas Hankel dos modos normais

\begin{tabular}{|c|c|c|}
\hline $\mathrm{n}^{\circ}$ do modo & $\gamma_{1}$ & $\gamma_{2}$ \\
\hline 1 & $2,2110^{4}$ & $8,1410^{3}$ \\
\hline 2 & $1,7710^{4}$ & $1,1610^{4}$ \\
\hline 3 & $5,8110^{4}$ & $3,6510^{3}$ \\
\hline 4 & $2,2310^{5}$ & $7,3910^{4}$ \\
\hline 5 & $1,5810^{5}$ & $4,9110^{4}$ \\
\hline 6 & $1,7310^{5}$ & $7,2010^{4}$ \\
\hline 7 & $1,6510^{5}$ & $7,8810^{4}$ \\
\hline
\end{tabular}

Tabela 6: Ordem de importância dos modos normais segundo o critério NH

\begin{tabular}{|c|c|c|}
\hline $\begin{array}{c}\text { Importância } \\
\text { segundo critério }\end{array}$ & $\begin{array}{c}\mathrm{n}^{-} \text {do } \\
\text { modo }\end{array}$ & $\begin{array}{c}\text { Parâmetro de } \\
\text { seleção } P_{\gamma m}\end{array}$ \\
\hline $1^{o}$ & 4 & $2,2310^{5}$ \\
\hline $2^{\underline{o}}$ & 6 & $1,7310^{5}$ \\
\hline $3^{o}$ & 7 & $1,6510^{5}$ \\
\hline $4^{o}$ & 5 & $1,5810^{5}$ \\
\hline $5^{\underline{o}}$ & 3 & $5,8110^{4}$ \\
\hline $6^{\underline{o}}$ & 1 & $2,2110^{4}$ \\
\hline $7^{o}$ & 2 & $1,7710^{4}$ \\
\hline
\end{tabular}

A tabela 6 mostra os parâmetros $P_{\gamma m}^{(k)}$, segundo o critério baseado nas normas Hankel dos modos normais (NH), conforme equação 28.

A tabela 7 mostra uma comparação da ordem de importância que cada critério de seleção deu à cada modo normal do modelo completo.

Observa-se certa concordância entre os critérios FN e AFN ao selecionarem os modos 1,2 e 3 como os mais indicados a serem preservados nos modelos reduzidos. Entretanto os mesmos critérios divergem ao determinar os modos que são bons candidatos à eliminação. Segundo o critério FN, os dois modos mais indicados para serem eliminados são 6 e 7, enquanto o critério AFN indica os modos 4 e 5.

Pode-se observar que os critérios FN e AFN divergem consideravelmente em relação ao critério NH. Como exemplo, os modos 1,2 e 3 são indicados pelos critérios FN e AFN como os mais significativos para a aproximar o comportamento dinâmico do sistema completo em um modelo reduzido. Enquanto que o critério NH classifica os mesmos modos como menos significativos para tal tarefa.

\subsection{Resultados dos modelos reduzidos}

A partir do modelo completo, 3 modelos de ordem reduzida foram construídos, cada um utilizando um dos 3 métodos de seleção descritos anteriormente (FN, AFN e NH). Cada modelo reduzido foi obtido através do truncamento dos 3 modos menos significativos para cada critério. 
Tabela 7: Comparação da ordem de importância dos modos segundo diferentes critérios

\begin{tabular}{|c|c|}
\hline $\begin{array}{c}\text { Critério } \\
\text { de seleção }\end{array}$ & $\begin{array}{c}\text { Ordem de importância } \\
\text { dos modos }\end{array}$ \\
\hline FN & $1,2,3,4,5,6,7$ \\
\hline AFN & $1,2,3,6,7,4,5$ \\
\hline NH & $4,6,7,5,3,1,2$ \\
\hline
\end{tabular}

A tabela 8 mostra os modos normais que foram preservados e quais foram truncados para cada modelo.

Tabela 8: Modos normais preservados e eliminados em cada modelo reduzido

\begin{tabular}{|c|c|c|}
\hline $\begin{array}{c}\text { Nome do } \\
\text { modelo }\end{array}$ & $\begin{array}{c}\text { modos } \\
\text { preservados }\end{array}$ & $\begin{array}{c}\text { modos } \\
\text { truncados }\end{array}$ \\
\hline completo & $1,2,3,4,5,6,7$ & - \\
\hline FN & $1,2,3,4$ & $5,6,7$ \\
\hline AFN & $1,2,3,6$ & $7,4,5$ \\
\hline NH & $4,6,7,5$ & $3,1,2$ \\
\hline
\end{tabular}

Para que a resposta transiente entre os modelos seja comparada, os mesmos foram submetidos à uma entrada degrau $u=(0 ; 0 ; 0 ; 0,1)^{T}$, que corresponde à um degrau de altura $0,1 \mathrm{~m}$ no pneu traseiro esquerdo.

A figura 5 mostra a força normal do pneu traseiro esquerdo $F_{4}(t)$ para os modelos original e os reduzidos.

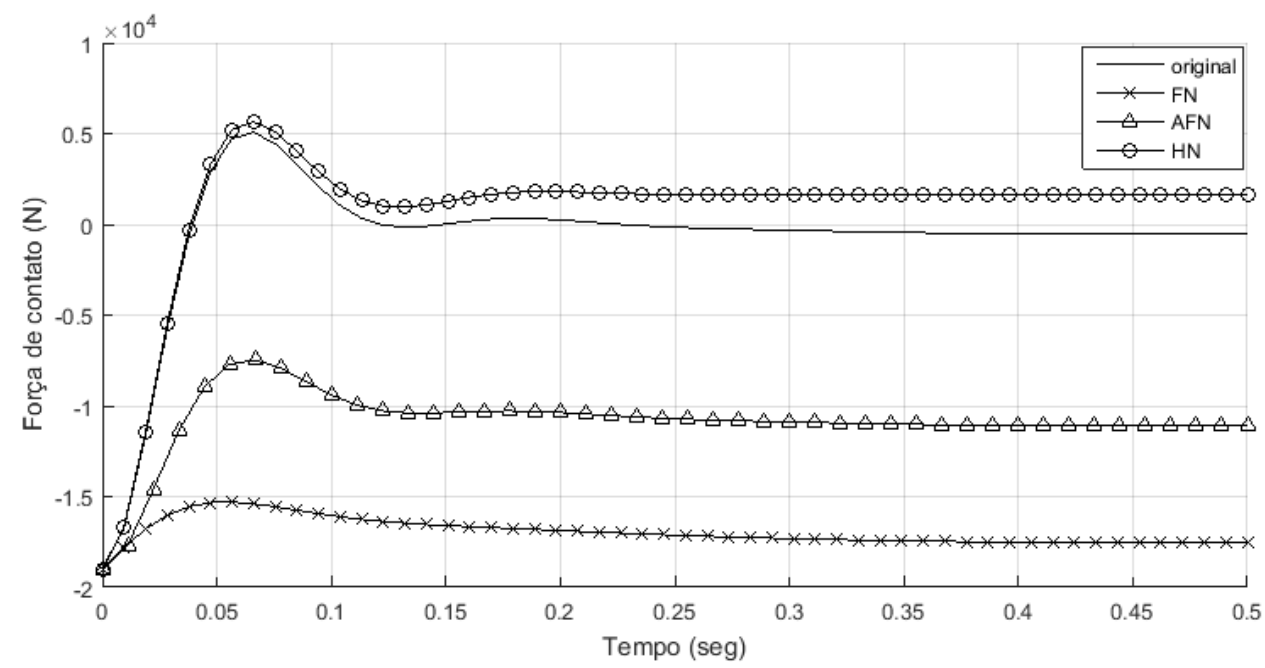

Figura 5: Resposta temporal Força F4 - entrada degrau

A figura 6 mostra o erro quadrático dos modelos reduzidos, de acordo com a Eq. 5. 


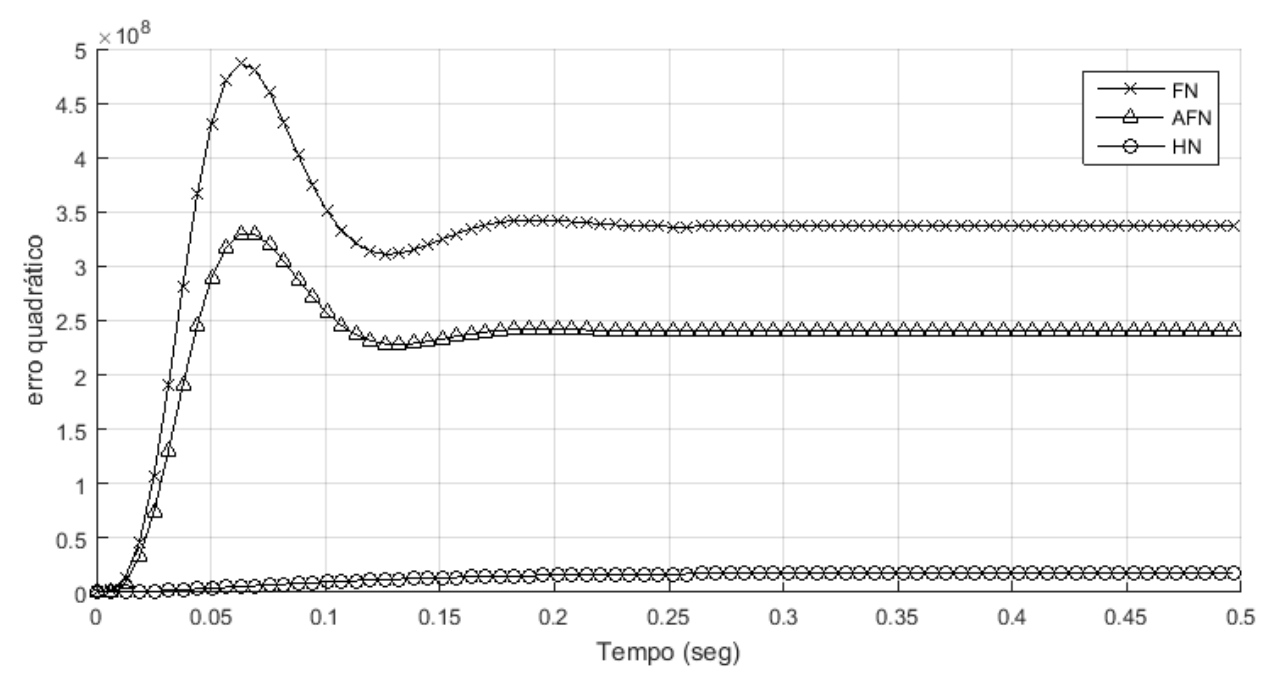

Figura 6: Resposta temporal erro quadrático - entrada degrau

Para avaliar a representatividade de cada modelo em função do número de modos truncados, os autores propõem a comparação através da norma $\mathcal{L}_{2}$ do erro dos modelos. A figura 7 mostra a norma $\mathcal{L}_{2}$ no intervalo $\left[\begin{array}{ll}0 & 0,5]\end{array}\right]$ do erro de cada modelo reduzido (submetidos à entrada degrau descrita acima) em função do número de modos truncados.

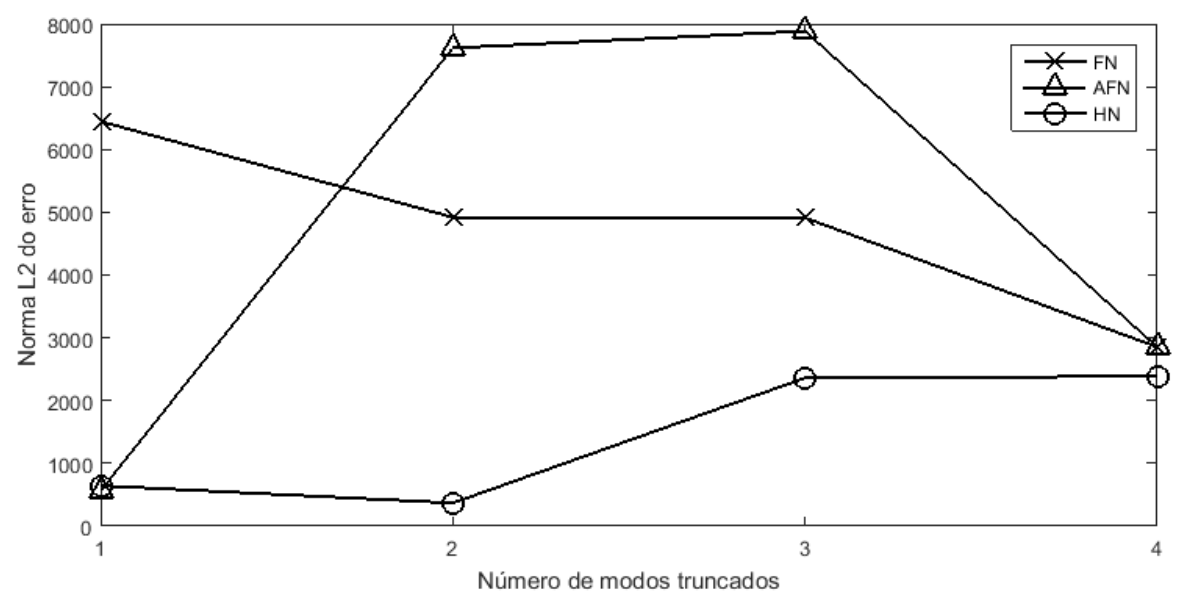

Figura 7: Norma $\mathcal{L}_{2}$ do erro em função do número de modos truncados

Baseando-se nas duas metricas de comparação apresentadas, erro quedrático e norma $\mathcal{L}_{2}$ do erro, os modelos obtidos com o critério de seleção baseado nas normas Hankel dos modos $(\mathrm{NH})$ tiveram melhor desempenho quando comparados a outros obtidos pelos demais critérios de seleção (FN e AFN). 


\section{CONCLUSÕES}

Os autores expuseram uma metodologia de redução da ordem de sistemas dinâmicos por truncamento na base modal. Foram calculados três modelos de ordem reduzida para determinação da dinâmica vertical de um veículo. Para cada modelo foi utilizado um critério diferente de seleção dos modos a serem truncados. Posteriormente os resultados dos modelos reduzidos foram comparados aos do modelo completo utilizando métricas que favorecem a avaliação objetiva de seus desempenhos como erro quadrático e norma $\mathcal{L}_{2}$.

Dos três modelos reduzidos calculados, o que apresentou melhores resultados (avaliados pelo erro quadrático e norma $\mathcal{L}_{2}$ do erro) foi o obtido pelo critério de seleção baseado nas normas Hankel dos modos normais.

\section{Referências}

[1] Gawronski, W. K. Dynamics and control of structures: A modal approach. Springer New York, 1998.

[2] Goldstein, H. Classical mechanics, vol. 4. Pearson Education India, 1962.

[3] Gregory, C. Reduction of large flexible spacecraft models using internal balancing theory. Journal of Guidance, Control, and Dynamics 7, 6 (1984), 725-732.

[4] Hirsch, M. W., Devaney, R. L., And Smale, S. Differential equations, dynamical systems, and linear algebra, vol. 60. Academic press, 1974.

[5] KIM, C., AND Ro, P. I. An accurate full car ride model using model reducing techniques. Journal of mechanical design 124, 4 (2002), 697-705.

[6] Mahala, K. M., Gadkari, P., Deb, A., ET Al. Mathematical models for designing vehicles for ride comfort. In ICORD 09: Proceedings of the 2nd International Conference on Research into Design, Bangalore, India 07.-09.01. 2009 (2009).

[7] Obinata, G., And Anderson, B. Model Reduction for Control System Design, vol. 1. Springer Verlag, 2001.

[8] Ogata, K., And Yang, Y. Modern control engineering, 4th ed. ed. Prentice-Hall Englewood Cliffs, 1970.

[9] Pernebo, L., And Silverman, L. Model reduction via balanced state space representations. Automatic Control, IEEE Transactions on 27, 2 (1982), 382-387.

[10] SoARes, M. E. Modos não lineares em sistemas discretizados pelo método de elementos finitos. PhD thesis, Escola Politécnica da Universidade de São Paulo, 1998.

[11] VARgA, A. On modal techniques for model reduction. Tech. rep., Technical Report TR R136-93, Institute of Robotics and System Dynamics, DLR Oberpfaffenhofen, PO Box 1116, D-82230 Wessling, Germany, 1993. 